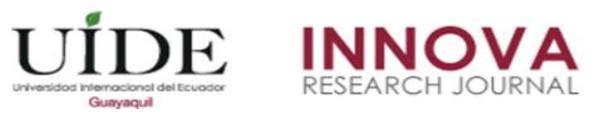

INNOVA Research Journal, ISSN 2477-9024

(Abril, 2017). Vol. 4, No.4 pp. 1.-11

DOI: https://doi.org/10.33890/innova.v2.n4.2017.143

URL: http://revistas.uide.edu.ec/index.php/innova/index

Correo: innova@uide.edu.ec

\title{
Beneficios de utilizar software BPM en los procesos de la facultad de ciencias administrativas de la universidad de Guayaquil
}

\section{Benefits of using BPM software in the processes of the faculty of administrative sciences of the university of Guayaquil}

Jorge Misael Merchán Riera

Christian Joseph Moreno Rodríguez

Melvin Leonardo López Franco

Universidad de Guayaquil, Ecuador

Autor para correspondencia: jorge.merchanr@ug.edu.ec, christian.morenorod@ug.edu.ec, melvin.lopezf@ug.edu.ec

Fecha de recepción: 13 de Enero de 2017 - Fecha de aceptación: 20 de Marzo de 2017

Resumen: La siguiente investigación tiene como objetivo general demostrar los beneficios de utilizar software BPM en los procesos administrativos y académicos de la Facultad de Ciencias Administrativas de la Universidad de Guayaquil, es interesante que al escuchar por vez primera sobre BPM parece ofrecer el mismo beneficio principal que la tecnología de aplicaciones empresariales tradicionales como ERP: aumento de la productividad de la fuerza de trabajo (como resultado de la racionalización de las operaciones comerciales y la automatización de tareas repetitivas). No obstante, las plataformas BPM van mucho más allá de un simple aumento en la eficiencia organizacional ya que ofrece capacidades de intercambio de conocimientos y colaboración integrada que contribuyen a una mejor toma de decisiones. A corto plazo, el BPM ayuda a las organizaciones a disminuir los costos y aumentar la eficiencia, lo cual puede significar mayor velocidad, organización y eficiencia para las instituciones universitarias. A largo plazo, utilizar software BPM ayuda a crear ventaja competitiva mejorando la agilidad de la organización. La tecnología de plataforma BPM puede hacer que cualquier organización incluyendo las universitarias -en los sectores público y privado- sea más eficiente, más ágil y más exitosa, sin que afecte mucho su presupuesto.

Palabras clave: software bpm; administración; manejo de procesos; universidad de guayaquil

Abstract: The following research has the general objective of demonstrating the benefits of using BPM software in the administrative and academic processes of the College of Administrative Sciences of the University of Guayaquil, it is interesting that the first listening about BPM, it seems to offer the same main benefit than traditional enterprise application technology such as ERP: Increased productivity of the workforce (as a result of streamlining business operations and automation of repetitive tasks). However, BPM platforms go far beyond a simple increase in organizational efficiency because it offers knowledge sharing and integrated collaboration capabilities that contribute to better decision making. In the short term, BPM helps organizations lower costs and increase efficiency, this can mean greater speed, organization and efficiency for university institutions. In the long term, BPM software helps create competitive advantage by improving the agility of the organization. The BPM platform technology can make any organization including universities - in the public and private sectors - more efficient, more agile and more successful, without affecting its budget in a relevant way. Key Words: bpm software; management; process management; university of guayaquil 


\section{Introducción}

El siguiente estudio describe los efectos positivos de utilizar software BPM en el manejo de los procesos y como estos podrían beneficiar a los procesos académicos y administrativos de la Facultad de Ciencias Administrativas de la Universidad de Guayaquil.

La gestión de procesos de negocio (BPM) permite a las organizaciones alinear las funciones internas con las necesidades de los clientes (en este caso serían los estudiantes) y ayuda al ejecutivo a determinar cómo dirigir, supervisar y medir los recursos de la organización.

El BPM, cuando es ejecutado correctamente por profesionales cualificados, tiene la capacidad de reducir costos, aumentar la eficiencia y la productividad y minimizar los errores y los riesgos, protegiendo y optimizando los recursos organizacionales.

Debido a que la gestión de procesos de negocio implica asignar y rastrear dichos recursos organizacionales, generalmente hay menos errores que en las organizaciones que no supervisan activamente esos recursos. Las organizaciones que siguen las mejores prácticas de BPM encontrarán que pueden reducir drásticamente dichos errores, aumentar la eficiencia y, en última instancia, aumentar la calidad de su servicio.

Los exámenes regulares de rendimiento pueden identificar posibles casos de ineficiencias, y las mejoras continuas del proceso pueden ayudar a resolverlas.

Otro beneficio del BPM es su capacidad para hacer cumplir medidas de seguridad y protección de información. Al documentar los procedimientos adecuados y exigir el cumplimiento total de los mismos, las organizaciones pueden ayudar a garantizar la seguridad del personal y proteger los activos de la organización -incluyendo recursos físicos e información confidencial- de robo, pérdida o uso indebido. Es por eso que los controles internos y las políticas organizacionales juegan un papel clave en la gestión de procesos de negocio.

Por otra parte, durante la investigación de campo se determinaron algunas carencias en la antes mencionada coordinación académica, dónde la muestra de empleados manifestó la necesidad de la implementación de nuevas tecnologías y software que les permitiera mantener un mejor control y seguimiento de los procesos inherentes a ella.

En este orden de ideas, la presente investigación busca mostrar las posibles alternativas que pudiesen implementarse en la Facultad de Ciencias Administrativas de la Universidad de Guayaquil con motivo de responder a esta necesidad hecha evidente por nuestros instrumentos de recolección de datos.

\section{Marco Teórico}

Según Nathaniel Palmer (2014) "Enabling the Digital Enterprise”, el Business Process Management (BPM) puede definirse como: "El Business Process Management (BPM) es una disciplina que involucra cualquier combinación de modelado, automatización, ejecución, control, medición y optimización de flujos de actividad empresarial, en apoyo de objetivos empresariales, sistemas, empleados, clientes y socios dentro y más allá de los límites de la empresa." 
Según Idalberto Chiavenato (2004) "Introducción a la Teoría General de la Administración”, Séptima Edición, McGraw-Hill Interamericana, Pág. 10. La administración puede definirse como: “...el proceso de planificar, organizar, dirigir, y controlar el uso de los recursos para lograr los objetivos organizacionales.”

De la misma manera para el autor Sabino Ayala Villegas (1996) "Gestión Empresarial”, Tomo I. La gestión empresarial o administración es considerada como: “...El proceso administrativo, que, implementado de forma sistemática, genera resultados favorables en la gestión de empresas, dicho proceso considera a la administración como la ejecutante de ciertas actividades llamadas funciones administrativas, Planeación, Organización, Dirección y Control."

Finalmente es importante que se defina teóricamente los conceptos de "cuello de botella" y "tiempos de respuesta" en los procesos administrativos, para ello recurrimos a Ángel Antonio Romero (2016) “¿Qué es el cuello de botella y cómo afecta a un proceso productivo?” que nos define ambos: "Decimos que en nuestra cadena de producción tenemos un cuello de botella cuando una fase de nuestro proceso productivo es más lenta que las demás y eso ralentiza nuestro proceso de producción en general. Dicho de otra manera, un cuello de botella es una restricción de la capacidad del sistema que nos produce una caída considerable de la eficiencia." "...Debido al cuello de botella, las etapas siguientes del proceso van a sufrir retrasos en forma de tiempos de parada no deseados, van a reducir la productividad y van a generar, por tanto, un aumento de los costes."

\section{Marco Metodológico}

Las técnicas investigativas utilizadas en el presente estudio son:

- Documentación de fuentes secundarias y terciarias.

- Selección de población y muestra en la Universidad de Guayaquil.

- Implementación de un cuestionario en la Facultad de Ciencias Administrativas de la Universidad de Guayaquil.

- Contraste entre la información recabada en la Facultad de Ciencias Administrativas de la Universidad de Guayaquil con las investigaciones del uso del software BPM en organizaciones

- Desarrollo de una conclusión dónde se detalle la importancia y beneficios del uso de software BPM para la mejora de procesos en la Facultad de Ciencias Administrativas de la Universidad de Guayaquil.

\section{Documentación de fuentes secundarias y terciarias.}

Para esta investigación usaremos un método de campo-documental. Para esto se revisaron diversas fuentes disponibles sobre estudios relacionados con el uso de software BPM en empresas, instituciones, y demás organizaciones a nivel mundial, uso que ha registrado beneficios en ellas y que de igual manera pudiesen afectar de manera positiva el manejo de procesos administrativos y académicos de la Facultad de Ciencias Administrativas de la 
Universidad de Guayaquil, habiendo anteriormente detectado una necesidad tras la aplicación de nuestro instrumento de recolección de datos.

Algunas de las investigaciones usadas como referencia para la presente investigación fueron: Jim Rudden (2007) Making the Case for BPM: A Benefits Checklist. Una investigación que se realizó para determinar los beneficios del software en el ámbito empresarial, de igual forma proporciona algunos ejemplos de cómo la tecnología BPM ha ayudado al incremento de la eficiencia organizacional.

Asimismo, fue tomado en consideración el estudio de Oracle Insight (2009) Building the Business Case for BPM. Un estudio que afirma que el software BPM (Business Process Management) puede ser utilizado como una tecnología para ayudar a hacer que los procesos prácticos sean consistentes y repetibles. La tecnología es una inversión que puede proporcionar un beneficio comercial inmenso a la organización; sin embargo, la investigación también nos habla de que el valor comercial tangible asociado con BPM generalmente no es bien entendido. Este estudio destaca el uso de la tecnología BPM como una forma de mejorar beneficiar la mejora de los procesos de negocio. Afirmando a su vez que la tecnología BPM también puede brindar beneficios que no pueden lograrse mediante técnicas tradicionales de mejora de procesos.

Finalmente nos referimos a la investigación llevada a cabo por la organización Capgemini (2012) Global Business Management Report. Esta investigación se basa en una exploración de las tendencias clave en el mercado global con respecto al uso de software BPM en organizaciones, de la misma manera también se enfoca en las dificultades que tiene algunas organizaciones para la implementación de estas tecnologías y como pueden socavarse para que puedan implementarse correctamente en dichas organizaciones.

\section{Selección de Población y Muestra}

Para la realización del análisis cuantitativo-estadístico, se tomó como muestra 250 de los 458 docentes que realizan actividades en los procesos académicos y administrativos de la Facultad de Ciencias Administrativas, se tomó como referencia la Facultad de Ciencias Administrativas puesto que es una de la facultades más numerosa en estudiantes y docentes en la Universidad de Guayaquil, y la temática de la investigación es afín a sus campos de estudio.

Cabe indicar que de acuerdo a las últimas disposiciones de las autoridades de Educación Superior el personal docente además de sus actividades como docentes también colaboran en los procesos académicas y administrativos.

\section{Instrumento de recolección de datos}

Para recolectar información de la Facultad de Ciencias Administrativas de Universidad de Guayaquil, se utilizó el instrumento la encuesta y la herramienta el cuestionario. Al contactarlos se les realizó las siguientes preguntas:

a) Sexo del participante.

b) Tiempo en su cargo.

c) ¿Considera usted que los procesos académico-administrativos son eficientes y rápidos? 
d) ¿Cuenta usted con una herramienta que le permita medir los tiempos de respuesta e identificar cuellos de botella?

e) Hasta los momentos, ¿cómo ha sido su experiencia al medir tiempos de respuesta e identificar cuellos de botella?

f) ¿Cree usted que mediante el uso de tecnologías se pudiesen mejorar los procesos?

g) ¿Le gustaría a usted contar con una herramienta informática que le permita conocer los tiempos de respuesta e identificar cuellos de botella?

h) ¿Sabe usted qué es el software BPM?

i) En caso de que lo conozca, ¿Cree usted que los procesos pudiesen agilizarse con el uso de software BPM?

\section{Resultados}

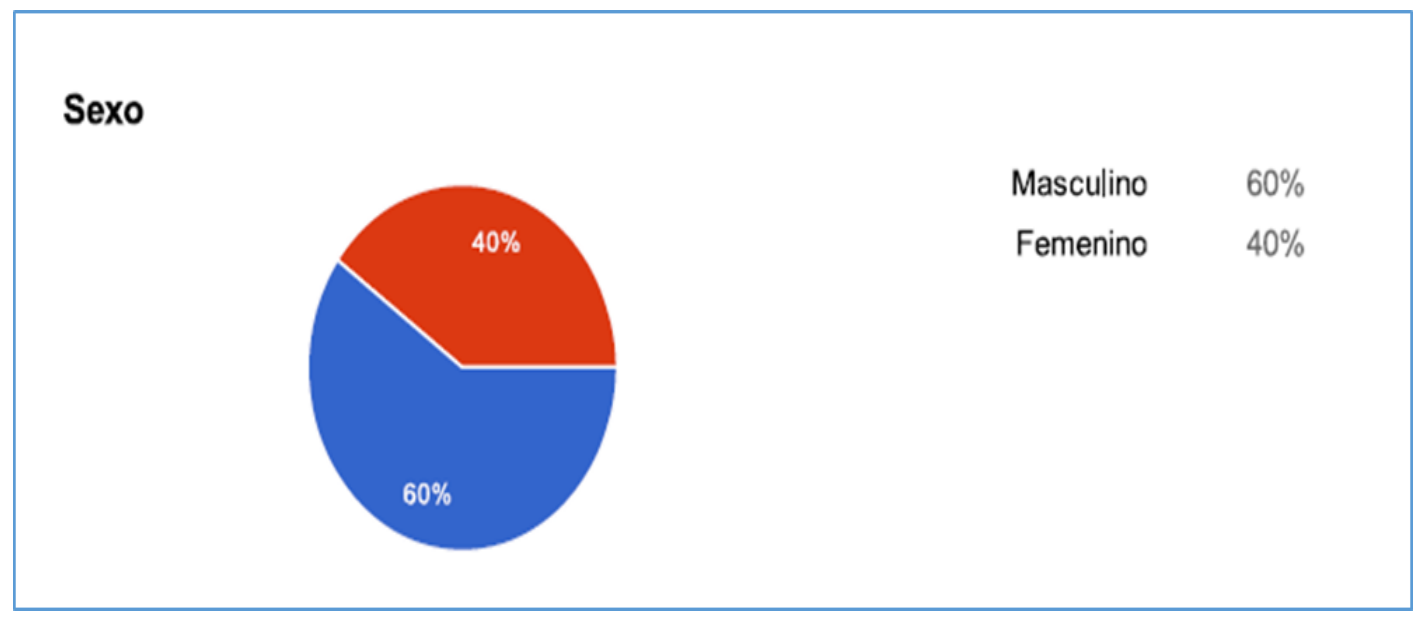

Figura No 1

Elaborado por los autores

Como se puede apreciar en la figura 1, la variable "A", la cual se refiere al sexo de las personas elegidas para la muestra (Masculino "M" -Azul- y femenino "F" -Rojo-), existe una mayoría masculina, aunque este dato no es tan incidente en esta investigación puede ser útil para una futura investigación en donde se tengan que demostrar habilidades y competencias por género.

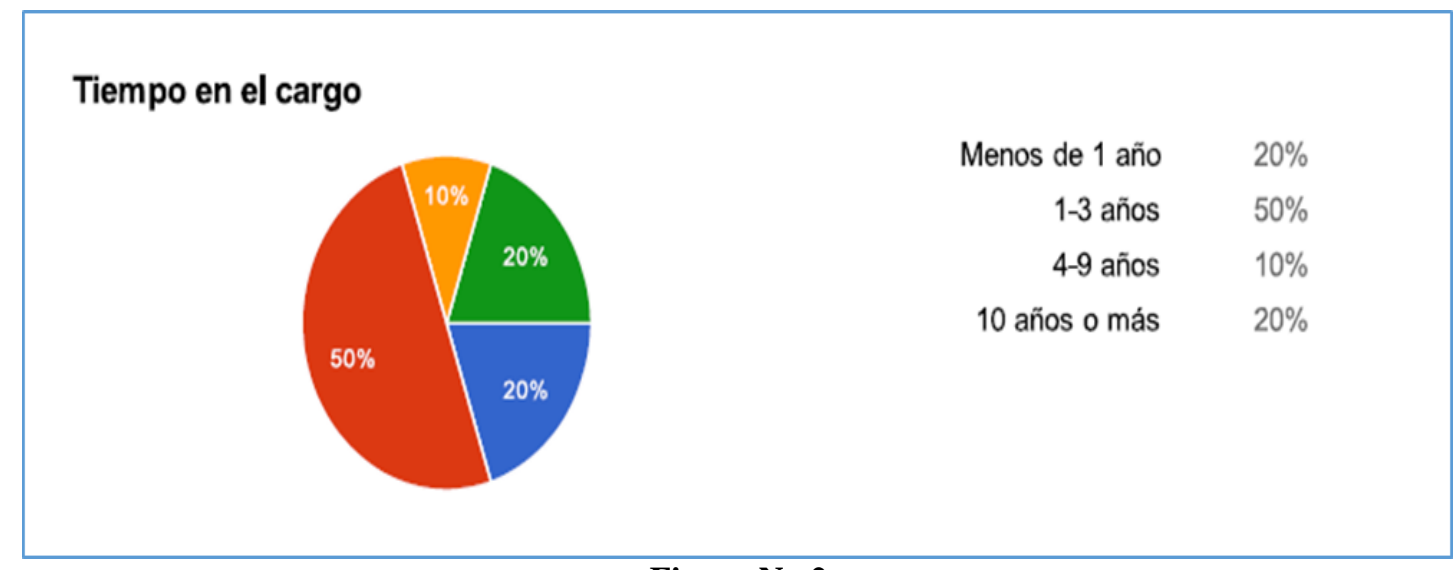

Figura No 2 


\section{Elaborado por los autores}

La variable "B", la cual se refiere al tiempo que las personas tienen ejerciendo su cargo actual en la Facultad de Ciencias Administrativas de la Universidad de Guayaquil. En la gráfica de la figura 2 podemos destacar que el valor más resaltante es la división de color rojo, la cual representa a la categoría "1-3 años" de la variable, lo cual nos indica que, de toda la muestra seleccionada la mayoría de las personas tienen de 1 a 3 años en su cargo es decir tienen un corto tiempo, es decir el personal es relativamente nuevo

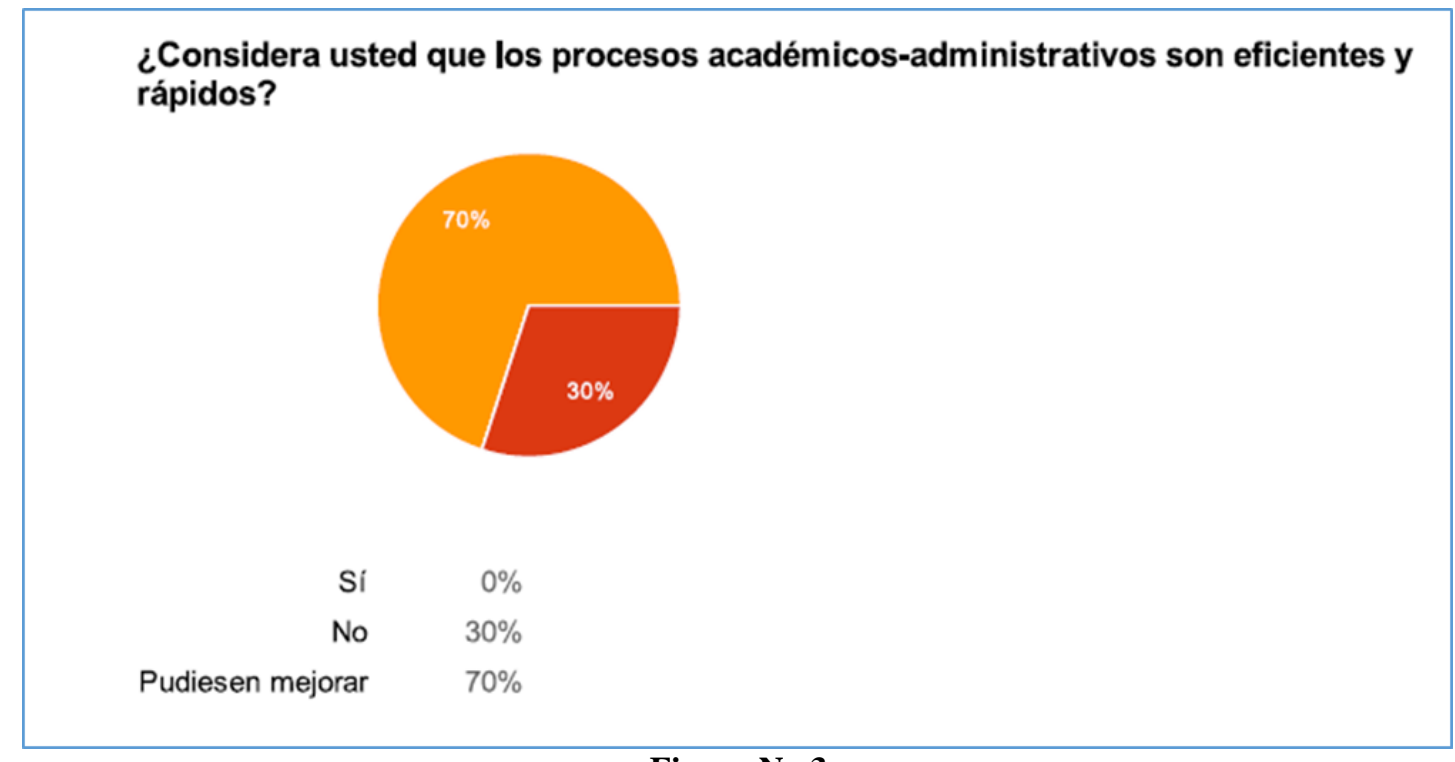

Figura No 3

Elaborado por los autores

A la variable "C", a la cual se llamó "Considera usted que los procesos académicosadministrativos son eficientes y rápidos" se le asignó un color por cada respuesta posible, en esta ocasión podemos claramente notar que la división de la gráfica más resaltante es de color naranja como se muestra en la Figura 3, este color representa a la categoría "Pudiese mejorar" de la variable en cuestión. Lo cual nos indica que la mayoría de los entrevistados consideran que hay espacio para la mejora de procesos, y esto a su vez se ve reflejado por la amplia porción que esta categoría representa en la circunferencia. En la investigación de Jim Rudden (2007) "Making the Case for BPM: A Benefits Checklist", se establece que el concepto de mejora continua es en el corazón de la propuesta de la importancia que tiene la aplicación del software BPM en los procesos administrativos. De hecho, Rudden también afirma que "...la capacidad de mejorar continuamente los procesos administrativos y obtener resultados positivos de forma consistente es lo que diferencia a este tipo de software de otros medios capaces de mejorar dichos procesos.”. Por lo tanto, al plantear la implementación del software BPM, es fundamental considerar no sólo las primeras iteraciones de procesos y qué beneficios podrían traer, sino que también es esencial considerar los beneficios que se irán agregando con el tiempo. 


\section{¿Cuenta usted con una herramienta que le permita medir los tiempos de respuesta e identificar los cuellos de botella en los procesos?}

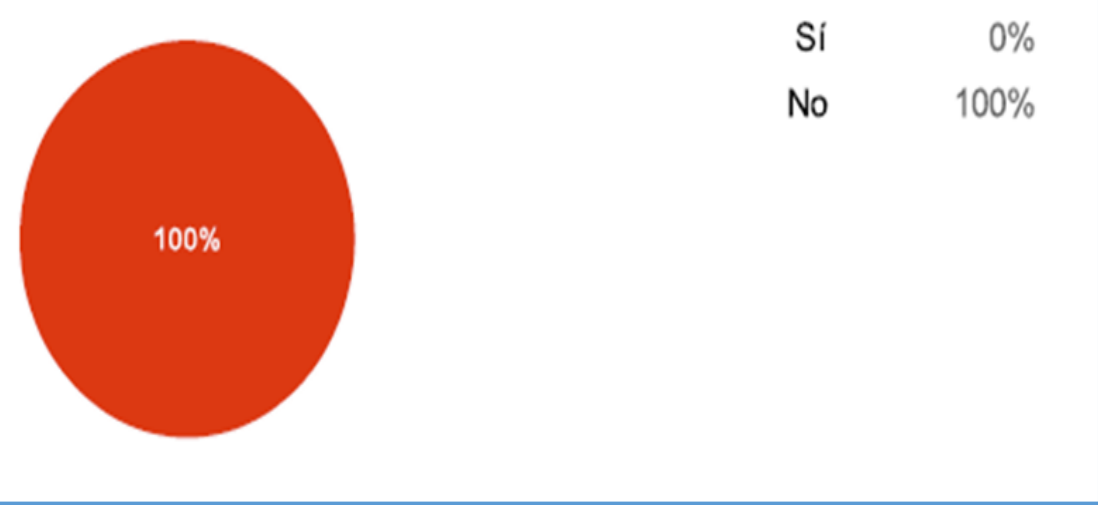

Figura No 4

Elaborado por los autores

Con respecto a la variable “ $D$ ”, la cual se llamó “¿Cuenta usted con una herramienta que le permita medir los tiempos de respuesta e identificar los cuellos de botella en los procesos? Se puede evidenciar en la Figura 4 que hay una gran necesidad dentro de la gestión de procesos de la Facultad de Ciencias Administrativas de la Universidad de Guayaquil, puesto que todas las personas seleccionadas como muestras admitieron carecer de una herramienta de cualquier índole para la identificación de los cuellos de botella y la medición de los tiempos de respuesta. En el estudio de Oracle Insight (2009) "Building the Business Case for BPM", se deja en claro que, en este mundo de cambio vertiginosos los administradores y/o gerentes necesitan información en tiempo real. Sin procesos automatizados, es muy difícil obtener información en tiempo real sobre la eficiencia en la ejecución de los procesos administrativos. Según el estudio “...El software BPM puede proporcionar la agilidad necesaria en un entorno empresarial de rápido cambio. La automatización de procesos es una forma evidente en la que el software BPM puede ayudar a una organización a ser más ágil en sus procesos administrativos. Los procesos organizados dentro del marco del software BPM están bien documentados con pasos claramente definidos". Además de estas ventajas, los cambios en los procesos existentes en la Facultad de Ciencias Administrativas se pueden hacer rápidamente dentro de un marco BPM porque sus bases teóricas ya son algo conocidas y tomadas en cuenta como una posible ayuda por parte del personal, como se evidenciará más adelante.

La variable "E", la cual se refiere a la experiencia que ha tenido la muestra seleccionada al momento de medir tiempos de respuesta e identificar cuellos de botella en los procesos se realizó de forma cualitativa, a modo de respuesta abierta para analizar las percepciones subjetivas de los entrevistados de la Facultad de Ciencias Administrativas de la Universidad de Guayaquil. En resumen las personas contestaron: "Sin resultados", "Complicada", "Terrible", "Imposible medir", etc.; debido a estas respuestas esta variable es crucial ya que evidencia muchas necesidades y carencias que pudiesen solucionarse con la implementación de algún software BPM. Tal y como afirma la organización Capgemini (2012) en su Global Business Management Report “... La aplicación de la tecnología BPM y su pensamiento automatiza procesos complejos, reduce el trabajo manual y elimina la duplicación de esfuerzos. El 74\% de 
los encuestados que implementaron una iniciativa de BPM con miras a reducir el trabajo manual reconocieron un impacto positivo en su negocio." Con la mejora de procesos brindada por software BPM la gestión académica y administrativa de la Facultad de Ciencias Administrativas de la Universidad de Guayaquil podría optimizarse y automatizar los métodos de medición de tiempos de respuesta e identificación de cuellos de botella, además de darle a todos sus empleados una visión completa de estos procesos y de cómo funcionan, proporcionando así un análisis más profundo y una visión más clara de estos.

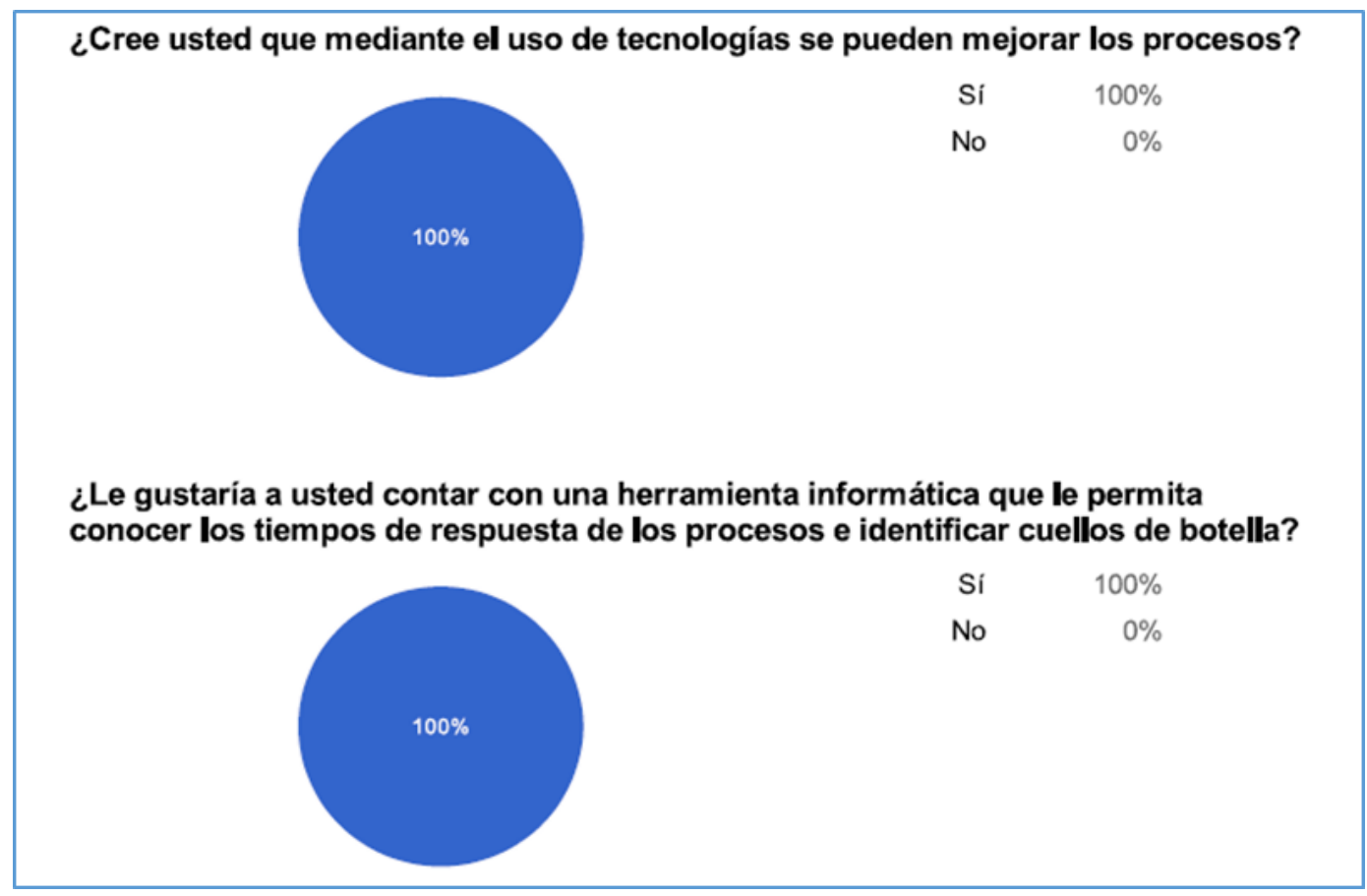

Figura No 5

Elaborado por los autores

Las variables " $F$ " y "G" que se muestran en la Figura 5 al ser de naturaleza similar se analizarán como una sola, en este caso la primera se refiere a la actitud de la muestra con respecto a la implementación de tecnología para mejorar los procesos académicos y administrativos de la Facultad de Ciencias Administrativas de la Universidad de Guayaquil, la segunda es la disposición de la misma muestra con respecto a su interés en contar con una herramienta informática que les permita medir tiempos de respuesta e identificar los cuellos de botella en los procesos. En ambas variables podemos observar que las personas tomadas en la muestra tienen una clara afinidad por el uso de software para la mejora de procesos, esta actitud es importante ya que facilitaría una posible implementación de un software BPM en los procesos. Nuevamente, en el estudio de Jim Rudden (2007) se pueden detallar algunos beneficios de la implementación de software BPM en las organizaciones, “...Los paquetes de BPM basados en estándares de la industria como TeamWorks tienen más probabilidades de implementarse con éxito utilizando los componentes de infraestructura existentes de cualquier organización." Esto nos indica que no hace falta tener una infraestructura de avanzada para el utilizar software BPM, y contando ya con al menos una infraestructura básica sumando al hecho de poseer empleados dispuestos a trabajar con dichas tecnologías, es evidente la importancia y la necesidad de implementarlas. 


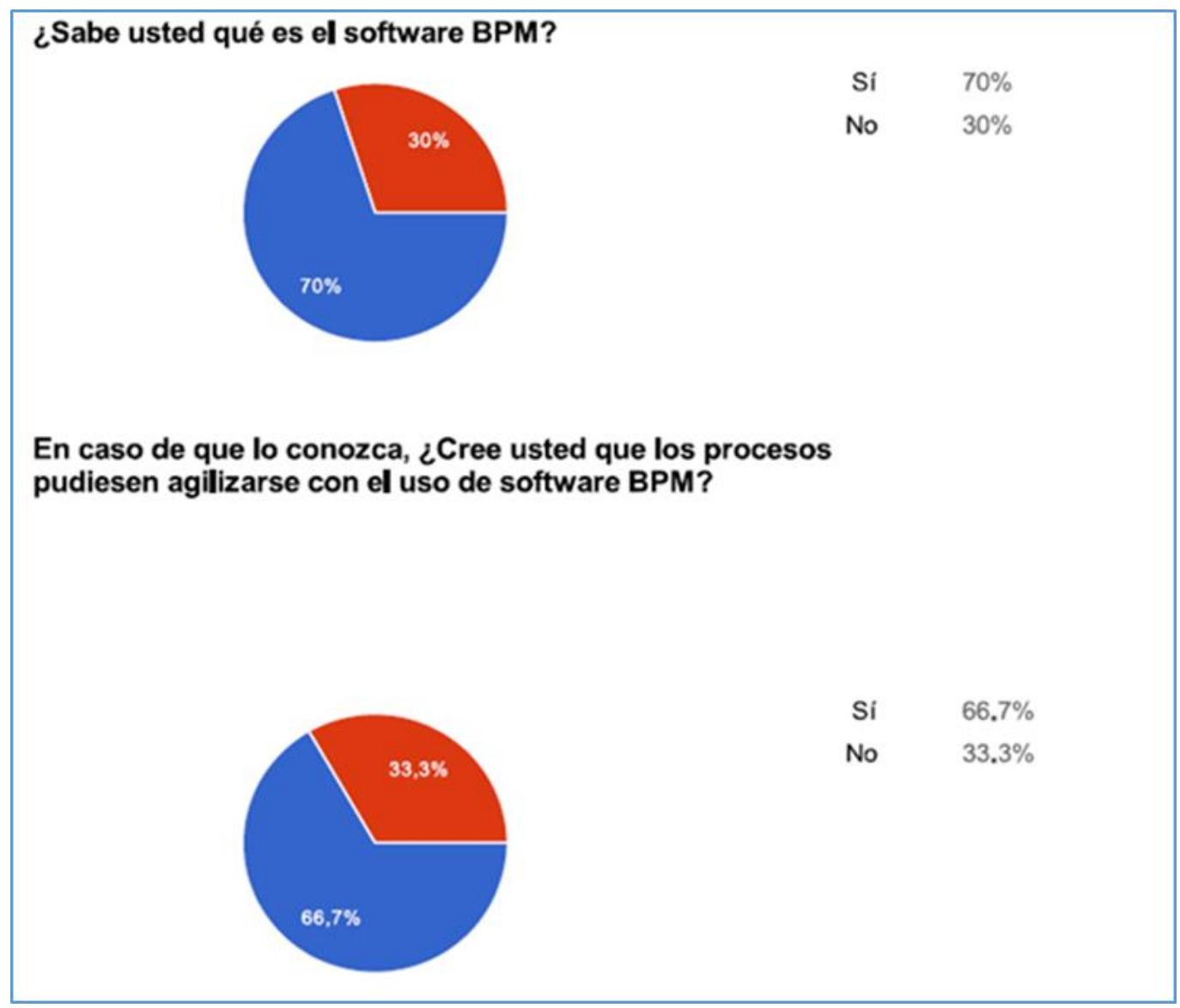

Figura No 6

Elaborado por los autores

Al igual que las dos anteriores variables, las variables " $\mathrm{H}$ " e "I" que se muestran en la Figura 6 se trabajarán de manera conjunta por la similitud de su naturaleza. La primera variable se refiere al conocimiento de la muestra sobre software BPM, la segunda variable es referente a, si en caso de conocer sobre el software BPM, los miembros de la muestra consideran que los procesos pudiesen agilizarse con la implementación de estos. Nuestras dos últimas variables son importantes, ya que nos indican que la muestra conoce algo sobre software BPM y consideran que su implementación podría traer beneficios al manejo de los procesos académicos y administrativos de la Facultad de Ciencias Administrativas de la Universidad de Guayaquil. Las tres investigaciones antes citadas hacen énfasis en cómo la implementación de software BPM podría traer agilidad en los procesos. Incluso, se podría afirmar que uno de los beneficios clave que ofrece el software BPM es la agilidad. Oracle Insight (2009) nos detalla aún más esto “...Los procesos automatizados dentro de un marco de BPM también ayudan a proporcionar velocidad al cumplimiento, así como transparencia y consistencia en la ejecución de los procesos administrativos.". Podemos entonces afirmar que los intereses de la muestra tomada están en concordancia con la teoría y las investigaciones sobre la implementación de software BPM en la agilización de procesos, lo cual es positivo al momento de considerar su implementación.

\section{Conclusión}


El beneficio del software BPM se ha visto evidenciado en variadas empresas en ámbitos diferentes, un buen ejemplo de esto puede ser la empresa minorista Carphone Warehouse que hizo público un aumento del $25 \%$ en sus índices de satisfacción al cliente después de invertir en software de gestión de procesos de negocio (BPM) para ayudar al personal a lidiar con las ventas, las consultas y las quejas. El minorista de teléfonos móviles, tuvo una facturación de 3.5 billones de libras durante el 2015, ha implementado el software BPM en más de 850 tiendas y tres centros de llamadas en el Reino Unido, y se está preparando para extender la tecnología por toda Europa. El sistema, llamado How2, proporciona a los trabajadores de las tiendas y al personal del centro de llamadas guías visuales simples de seguir, que cubren miles de consultas posibles por parte de los clientes.

Aparte de este caso concreto hay muchos programas informáticos de BPM disponibles que pudiesen ser útiles para las empresas y organizaciones, tales como:

Zoho Creator.- Cuando cada proceso de negocio es único y diferente el uno del otro, no puede haber un solo software que los resuelva a todos. Por esta razón más de 4 millones de usuarios en todo el mundo han construido aplicaciones personalizadas BPM para administrar sus procesos empresariales únicos. Zoho Creator proporciona herramientas esenciales y toda la ayuda que necesitan las organizaciones para crear aplicaciones personalizadas; Todo hecho por ellas mismas, independientemente de la experiencia técnica de los gerentes.

Process Street.- Process Street es una forma sencilla de gestionar los flujos de trabajo de los equipos de trabajo en las organizaciones, los procesos recurrentes (BPM) y los procedimientos operativos estándar (SOP).

QuickBase.- Crea aplicaciones que coincidan exactamente con los procesos únicos de cada organización, sin necesidad de código.

ProcessGene BPM Suite.- Una completa suite de software BPM, diseñada para organizaciones multi-subsidiarias.

Timereaction.- Flujogramas, conversaciones y archivos en un solo lugar, para que las organizaciones logren encontrar la información que necesitan, haciendo más, con menos esfuerzo.

Todas las antes mencionadas aplicaciones de software BPM están siendo utilizadas actualmente por empresas y organizaciones alrededor de todo el mundo, facilitando y agilizando los procesos y permitiendo un incremento significativo en la eficiencia de estas organizaciones, es evidenciable que si este software ha dado tantos dividendos en organizaciones de otros ámbitos, no hay duda de que también podría ser un aliado importante en la mejora de los procesos en la Facultad de Ciencias Administrativas de la Universidad de Guayaquil.

Al implementar un software BPM se proporciona la plataforma que necesita la Facultad de Ciencias Administrativas de la Universidad de Guayaquil para poder cambiar sus procesos administrativos y académicos -de una forma más ágil y controlada que con cualquier otra opcióneliminando en gran medida las posibilidades de errores o demoras, adicional a esto también se 
incluye la capacidad de cambiar un proceso para solucionar eventos imprevistos, sin que los tiempos de respuesta se vean afectados de manera negativa.

El uso de software BPM es una estrategia tecnológica que aporta beneficios a la organización al afectar tanto las líneas superiores como las inferiores de la misma. Sin embargo, estos beneficios tienen que ser cuantificados para que la organización sea capaz de demostrar en específico cuales procesos han mejorado tras su implementación, y eso es posible gracias a la naturaleza del software BPM. Esta tecnología no sólo traerá beneficios cuantificables para los procesos académicos y administrativos de la Facultad de Ciencias Administrativas de la Universidad de Guayaquil, sino que lo hará sin un aumento considerable en su inversión de recursos actual. Muchas empresas ya han comenzado a ver retornos sustanciales de sus proyectos de BPM, ya es tiempo de que las entidades educativas públicas también se beneficien de la automatización y simplificación de los procesos académicos y administrativos por medio del uso de tecnología.

\section{Bibliografía}

Ángel Antonio Romero (2016) “¿Qué es el cuello de botella y cómo afecta a un proceso Productivo?". Obtenido de http:/queaprendemoshoy.com/que-es-el-cuello-de-botella-ycomo-afecta-a-un-proceso-productivo/

Capgemini (2012) “Global Business Process Management Report”. Obtenido de https://www.capgemini.com/resource-fileaccess/resource/pdf/Global_Business_Process_ Management_Report.pdf

Idalberto Chiavenato (2004) "Introducción a la Teoría General de la Administración”, Séptima Edición, McGraw-Hill Interamericana, Pág. 10.

Jim Rudden (2007) "Making the Case for BPM: A Benefits Checklist”. Business Process Trends

Nathaniel Palmer (2014) “Enabling the Digital Enterprise”, Future Strategies Inc.

Oracle Insight (2009) "Building the Business Case for BPM". Oracle Corporation.

Sabino Ayala Villegas (1996) “Gestión Empresarial”, Tomo I., Graf. Castillo. 